\title{
A Smartphone Self-Management Program to Support Oral Chemotherapy Adherence in Young and Adult Cancer Patients: Design and Development
}

\author{
Skrabal Ross, X.12, Gunn, K.M ${ }^{1}$, Patterson, $\mathrm{P}^{3}$., Olver, I. ${ }^{4}$
}

University of South Australia, School of Health Sciences, ${ }^{2}$ Cancer Research Institute, University of South Australia, ${ }^{3}$ University of Sydney, ${ }^{4}$ University of Adelaide

\section{Background}

People diagnosed with cancer are responsible for self-administering oral chemotherapy under limited hospital monitoring but rates of adherence to oral chemotherapy in adults can be as low as $46 \%{ }^{1}$. There is also evidence of low medication adherence among adolescents and young Adults (AYA) with other chronic diseases ${ }^{2}$. Mobile phone-based interventions seem to increase medication adherence in other chronic diseases, but more evidence on whether these types of interventions support oral chemotherapy adherence is needed. This study describes the research-based process of development of a novel intervention to support young and adult cancer patients in taking their oral chemotherapy as prescribed by the doctor

\section{Main Reasons for Oral Chemotherapy Non- Adherence and Behavioral Change Strategies}

A Methodical database search of Medline, Embase, Emcare and PsychINFO was conducted to find systematic reviews on determinants of non-adherence to oral chemotherapy published between $01 / 2010$ and $03 / 2018$. More recent evidence to this review was provided by a second database search on research-based studies on reasons for oral chemotherapy/oral hormonal therapy nonpublished between 06/2015 and 03/2018. Five studies were incorporated into the review. ${ }^{15678}$

Three main reasons for non-adherence to oral chemotherapy (that had the potential to be modified by a mobile phone-based intervention) were identified: 1)forgetfulness, 2)side-effects and 3)poor knowledge about oral chemotherapy.

In depth analysis of the studies included in the review revealed behavior change strategies with the potential to positively affect the three main reasons for oral chemotherapy non-adherence: 1 ) text message medication intake reminders, 2,3) information about oral chemotherapy and side-effect management.

\section{Methods}

Based on Whitakker et al.'s framework for the development and evaluation of mhealth interventions ${ }^{3}$, the first two steps in the design process

(conceptualization and formative research) are described. An extensive literature review on the three main reasons for oral chemotherapy non-adherence, susceptible to be modified by a mobile phone-based intervention and strategies to positively affect those factors was conducted followed by a scoping review to explore what was known about available oral chemotherapy adherenceenhancing interventions delivered via mobile phones, the reported utility of the interventions as well as opportunities for future research ${ }^{4}$. Formative research consisted of a qualitative study that aimed to explore the preferences of people affected by cancer on the structure of elements of a mobile phone-based selfmanagement program focused on enhancing adherence to oral chemotherapy

\section{Gaps in Literature About Mobile Phone-Based Interventions to Increase Adherence to Oral Chemotherapy}

The scoping review methodological framework used in this review was outlined by Arksey and O'Malley'. Five studies describing four interventions were included in the review ${ }^{4}$. The study found a lack of evidence on the use of mobile phones in interventions to increase oral chemotherapy adherence but the existing interventions showed to be highly acceptable and useful to cancer patients. Available interventions were delivered through two mobile phone platforms: text messages, mobile phone apps or a combination of both platforms.

None of the interventions focused on supporting oral chemotherapy adherence in AYA. The engagement of stakeholders and the use of a design framework were identified as being important elements in the design process.
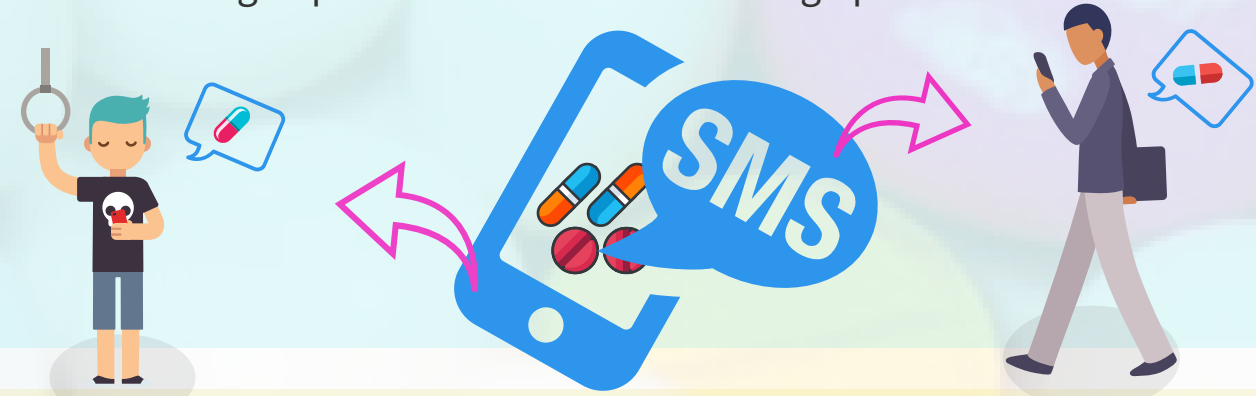

\section{Oral Chemotherapy Users' Preferences About the Structure of Elements in a Smartphone-Based Self-Management Program}

A qualitative study that aimed to explore the preferences of people affected by cancer on the structure of elements of a mobile phone-based selfmanagement program focused on enhancing adherence to oral chemotherapy was conducted. Nine oral chemotherapy users (20-71 y/o), $78 \%$ male, $22 \%$ female, treated in South Australian' hospitals completed semi-structured interviews. Data was analyzed using a Thematic Analysis approach. Common themes were identified which informed the elements of the program such as: mobile phone platform for intervention delivery, content and frequency of text message reminders, personalization, directionality and frequency of delivery of information about side-effects and oral chemotherapy.

\section{Conclusions}

A novel smartphone text message-based self-management intervention to support AYA and adult oral chemotherapy users in taking their drugs as prescribed was developed. A multi-site proof-of-concept trial of the tool is currently underway at six Australian hospitals. The design process described in this study may serve as a framework for the design of future studies that aim to support adherence to oral chemotherapy in both young and adult people affected by cancer.

\section{References}

. Kyngas, H. (2000). Compliance of adolescents with chronic disease. J Clin Nurs, 9(4), 549-556.

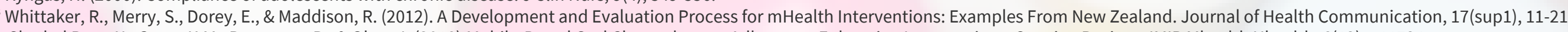
Skrabal Ross, X., Gunn, K.M., Patterson, P., \& Olver, I. (2018).Mobile-Based Oral Chemotherapy Adherence-Enhancing Interventions: Scoping Review. JMIR Mhealth Uhealth, 6(12), e11724. Corrente , J. (2016). Oral Chemotherapy, A Tough Pill To Swallow: A Systematic Review. Retrieved from

http://www.webcitation.org/queryrl=https\%3A\%2F\%2Fpdfs.semanticscholar.org\%2F0796\%2F284743b4f4e656705e26aa02e9731e4daed0.pdf\&date=2019-03-09

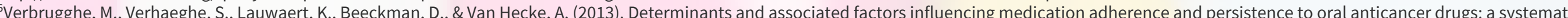
eview. Cancer Treat Rev, 39(6), 610-621.

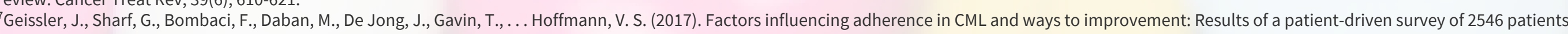
in 63 countries. J Cancer Res Clin Oncol, 143(7), 1167-1176.

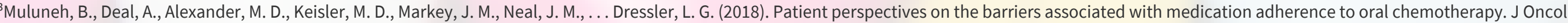
Pharm Pract, 24(2), 98-109. 